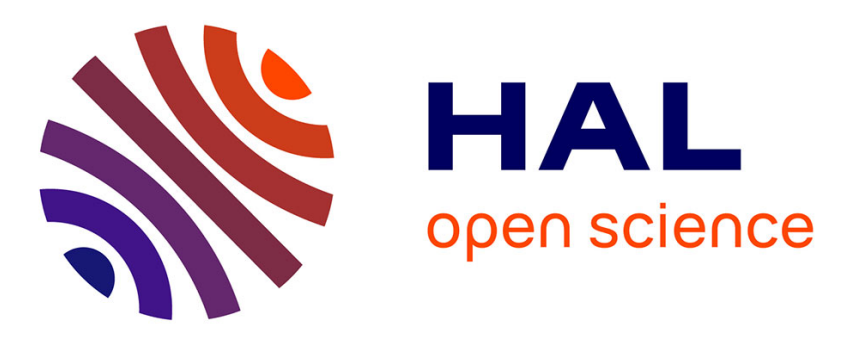

\title{
Enhancing the Driver Awareness of Pedestrian using Augmented Reality Cues
}

\author{
Minh Tien Phan, Indira Thouvenin, Vincent Frémont
}

\section{To cite this version:}

Minh Tien Phan, Indira Thouvenin, Vincent Frémont. Enhancing the Driver Awareness of Pedestrian using Augmented Reality Cues. 2016 IEEE 19th International Conference on Intelligent Transportation Systems (ITSC 2016), Nov 2016, Rio de Janeiro, Brazil. pp.1298-1304. hal-01398344

\section{HAL Id: hal-01398344 \\ https://hal.science/hal-01398344}

Submitted on 17 Nov 2016

HAL is a multi-disciplinary open access archive for the deposit and dissemination of scientific research documents, whether they are published or not. The documents may come from teaching and research institutions in France or abroad, or from public or private research centers.
L'archive ouverte pluridisciplinaire HAL, est destinée au dépôt et à la diffusion de documents scientifiques de niveau recherche, publiés ou non, émanant des établissements d'enseignement et de recherche français ou étrangers, des laboratoires publics ou privés. 


\title{
Enhancing the Driver Awareness of Pedestrian using Augmented Reality Cues
}

\author{
Minh Tien Phan, Indira Thouvenin, Vincent Frémont
}

\begin{abstract}
Pedestrian accident is a serious problem for the society. Pedestrian Collision Warning Systems (PCWS) are proposed to detect the presence of pedestrians and to warn the driver about the potential dangers. However, their interfaces associated with ambiguous alerts can distract drivers and create more dangers. On the other hand, Augmented Reality (AR) with Head-Up Display (HUD) interfaces have recently attracted the attention in the field of automotive research as they can maintain driver's gaze on the road. In this paper, we design a new PCWS with the AR cues and propose an experimental to evaluate the AR cues by assessing the driver's awareness of a pedestrian. At this stage, a fixed-based driving simulator is used for the study. Twenty five healthy middle-aged licensed drivers participate in the experiment. A car following task is proposed as the main driving task. Three levels of the driver's awareness of a pedestrian: the perception level, the vigilance level and the anticipation level are assessed through the observable outcomes. The results show that the proposed AR cues can enhance the driver's awareness of a pedestrian in all the three levels.
\end{abstract}

Index Terms-Augmented reality, Driver Behaviors; Pedestrian Safety; Situation Awareness; Driving Simulation;

\section{INTRODUCTION}

$\mathbf{I}$ $\mathrm{N}$ the domain of Advanced Driver Assistance Systems (ADAS), a close coupling between vehicle and driver can be achieved with active interfaces. Recently, several car manufacturers proposed many developments and commercialization plans about HUDs. This technology creates a new way for interactions between the driver and the vehicle. The HUDs can offer drivers various information related to safety and convenience such as velocity, navigation information, warning messages, etc. [5], [22], [8]. The HUD reduces the number and the duration of the driver's sight deviations from the road, by projecting the required information directly into the driver's line of sight. This allows drivers to receive information without lowering their gaze, thus avoiding attention gaps when taking their eyes off the road to look down at the driving information [7], [16]. Moreover, by highlighting important objects or regions, the AR cues help enhancing the visibility of some important elements in the road scene such as obstacles or pedestrians. It also helps the driver to do the right actions to avoid potential dangers.

In particular, pedestrians are known as the most vulnerable road users, whilst also being the most difficult to observe both in day and in night conditions [28]. Nowadays, the PCWS are

Phan Minh Tien, Indira Thouvenin, Vincent Frémont Heudiasyc Laboratory, University of Technology of Compiegne, France, e-mail: minh.phan@hds.utc.fr. able to detect the presence of pedestrians with high accuracy. Then, they can alert the driver in case of possible accidents across the beeps and sounds from a small interface posed on the dashboard [19]. However, the beeps and sound are only helpful if drivers know what sound means or if the sound occurs in conjunction with a visual cue [9]. We argue that the AR cues can give benefits in this kind of application. Therefore, the first objective of this paper is to propose an augmented reality assistance system that can enhance the driver's awareness of a pedestrian.

To the best of our knowledge, the work of Fukagawa et al. [13] is the only previous work that described the driver's awareness of a pedestrian. The authors considered the driver to be aware of a pedestrian when there was no accident in that situation. In this study, we propose a definition of Driver's Awareness of a Pedestrian (DAP) based on the Situation Awareness (SA) concept. In [11], SA is defined with three levels (perception, comprehension, projection) that describe how the operators maintain state and future state information of elements in their environment. In the situation where a pedestrian appears in front of the vehicle, the DAP is defined with three levels: The perception level, the vigilance level and the anticipation level (see Fig. 1). The perception level can be related to the moment and the period when the driver figures out there is a pedestrian on road. The vigilance level is the ability of the driver to sustain attention to the pedestrian and his estimation of the possibility that the pedestrian can cross the road. The anticipation level consists of right actions that driver chooses to do in that situation such as stopping, passing by, slowing down or speeding up, etc.

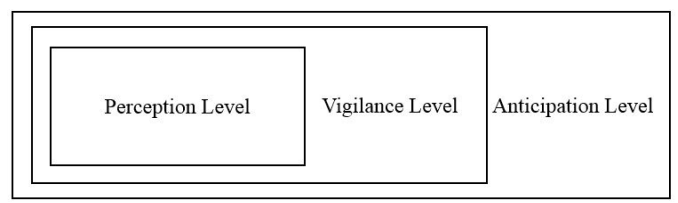

Figure 1: Driver Awareness of Pedestrian (DAP) definition

The second objective of this paper is to propose an experimental protocol that allows to evaluate implicitly three levels of the driver's awareness of a pedestrian. Finally, by comparing the DAP between the drivings with and without AR cues, we can determine costs and benefits of the cues in the critical situation with pedestrians.

Overall, this paper is organized as follows: Part II presents some related AR applications in automobile and presents our 
proposed AR-PCW system. Part III presents some related work in driver's awareness assessment followed by our experiment design that aims to evaluate the AR cues by assessing the driver's awareness of a pedestrian. The experimental results are showed and discussed in Part IV. Finally, Part $\mathrm{V}$ gives a conclusion and presents some future works of the study.

\section{Proposed System}

\section{A. HUD Prototype and Augmented Reality Cues}

According to our knowledge of industrial wind-shield manufacturing, only a small windshield zone in front of the driver can be dedicated to the AR zone [6]. Therefore, we propose to study a HUD combiner that allows to project the virtual images only on the center part of the windshield (see Fig. 2b). This part cover about fifteen degree of the driver's field of view

Concerning the AR cues, we consider to use two cues. Firstly, we propose a conformal cue which is the bounding box at the pedestrian position (see Fig. 2a). This cue is expected to remind that the pedestrian is vulnerable and can cross the road at any time. Secondly, we propose a nonconformal cue which is the warning panel. The panel is displayed at the bottom left of the HUD. In case the system detects that the situation becomes critical, a yellow pedestrian warning panel is displayed at the left corner of the HUD. This cue warns directly driver from the potential accident to the pedestrian. The yellow color is chosen for two cues, to convey a warning rather than an immediate threat [4], [14].

Indeed, the non-conformal cues are displayed at a predefined depth (usually2.5m to $4 \mathrm{~m}$ ) on the road in front of the driver. They are typically used to display the speed or other vehicle information [3]. The advantage of the non-conformal displaying is that the driver knows exactly where the cue is and gets used to it. However, it can create occlusion with the traffic elements such as precedent cars or obstacles. On the contrary, a conformal display is represented by the virtual imagery that is overlaid on the traffic elements . The image is optically superimposed on the objects it augments [15], [27]. In the driving context, this type of cue has advantage to enhance the visibility of the obstacles. In [24], the authors proposed also to use the bounding box as a conformal aid to highlight the hazards and to direct the driver attention to them (see Fig. 2b). The authors found that the static bounding box was actually related to longer reaction times than using no cues. In [25], the authors explored the use of bounding boxes for elderly drivers and found that they improved detection of hazardous object under low visibility, while not interfering with the detection of non-hazardous objects.

\section{B. Decision Module}

The decision module of the AR-PCW system consists of defining the rules to execute the visual cues (the bounding box and the warning panel). The Bounding Box (BB) is displayed whenever a pedestrian is detected in front of the driver.
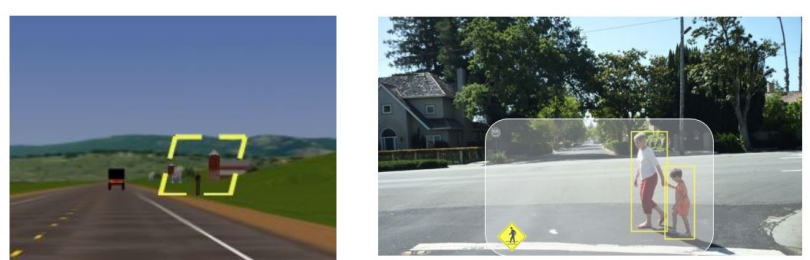

(a) Example of a pedestrian(b) The proposed visual cues for Pedeshighlighting [24] trian Collision Warning Systems.

Figure 2: Augmented reality cues for pedestrian collision warning systems.

Otherwise, the warning panel is displayed only when the situation is detected to be critical. At first stage, we consider the Time-To-Collision (TTC) and the distance to the pedestrian $(d)$ to determine the moment when the warning panel is displayed $\left(t_{W P}\right)$. By neglecting the pedestrian speed, the $T T C$ is calculated as follows:

$$
T T C=\frac{d}{v_{v e h}}
$$

where $v_{v e h}$ is the vehicle speed.

Now, let consider $T T C_{\text {critical }}$ and $d_{\text {critical }}$, which are the critical Time to Collision and the critical distance to pedestrian. The warning panel display moment $t_{W P}$ is computed as follows:

$$
t_{W P}=\min \left(t\left(T T C_{\text {critical }}\right), t\left(d_{\text {critical }}\right)\right)
$$

The choice of $T T C_{\text {critical }}$ is based on the cumulative human response time distribution. This distribution means the percentage of population that is able to react to a potential collision. To summarize, the larger the response time, the bigger the percentage of population to react on time to the warning. But a larger response time is expected to lead to a worse performance of the warning because it can become annoying to the driver. This distribution is $1.0 \mathrm{~s}, 1.6 \mathrm{~s}$, and $2.0 \mathrm{~s}$, corresponding to $45 \%, 80 \%$, and $90 \%$ of the population, respectively [2], [18]. In order to correspond to a large population, our proposed system is aimed to correspond to $90 \%$ of population, so the $T T C_{\text {critical }}$ is chosen to be $2 s$.

On the other hand, the critical-Distance $\left(d_{\text {critical }}\right)$ plays the role of a safety net to determine the critical situation. In some cases where the vehicle approaches the pedestrian with a low speed, the $T T C_{\text {critical }}$ is met when the vehicle is too close to the pedestrian. For example, when the vehicle speed $\left(v_{\text {veh }}\right)$ is about $20 \mathrm{~km} / \mathrm{h}(5.5 \mathrm{~m} / \mathrm{s})$ and the $T T C_{\text {critical }}$ is $2 s$, the distance is equal to $11 \mathrm{~m}$. It is already too close to the pedestrian. The $d_{\text {critical }}$ should be taken into account first. The $d_{\text {critical }}$ is then chosen to be $16.6 \mathrm{~m}$ in the cases when the speed is lower than $30 \mathrm{~km} / \mathrm{h}$. Finally, the moment to display the warning panel $\left(t_{W P}\right)$ are given as follows:

$$
t_{W P}=\min (t(T T C=2), t(d=16.6))
$$




\section{EXPERIMENTS DESIGN}

\section{A. Related Works}

A technique called SAGAT (Situation Awareness Global Assessment Technique) is a global tool developed to access Situation Awareness (SA) of the cockpit pilots. Endsley [12] lays out the criteria for SA measurement which has subsequently become one of the standard instruments. Here, participants are intermittently queried, in the middle of a dynamic simulation, about the values of various state parameters in the process under supervision. In the driving context, the SAGAT technique has also been used to assess the driver's SA at the intersections. In [23], the authors have created four critical scenarios at the intersections and provided a questionnaire to evaluate their focus of attention before accident. This technique is easy to use when one can build a set of queries about the situation to assess the driver SA in that specific situation. However, this technique is intrusive because the driver has to stop driving to answer the questions [1]. The participants may need to recall specific information on the driving environment from their earlier performance.

As another approach, the loss of SA can be inferred from changes in performance on tasks for which good SA is essential. For example, the freeway driver who pulls into another lane in front of an overtaking car can be inferred to have poor SA. This would be an example of an implicit SA measure. In [17], the authors investigated the effects of adaptive cruise control (ACC) and mobile phone use on driver SA and performance in a lead-car following task primarily involving operational behaviors. Their results revealed that SA improved with the use of ACC and decreased with use of the mobile phone under normal driving conditions. In [20], the authors have used the second task in order to study the performance of the driver and to assess the Situation Awareness. At predetermined road points, the choice to perform an additional task was proposed to the driver. The possibility of accepting and rejecting the second task was then given either just before a critical situation or in a noncritical situation. The driver's situation awareness is scored if the driver does not accept the secondary task in critical situations. The result showed that the driver rejected more secondary tasks in critical compared than in the non-critical situations. This technique seems more natural to assess the driver's SA. However, the use of a distraction is inappropriate in driving. The accident happened because of the distraction and not the driver's low SA. Moreover, our goal is to evaluate the visual cues which are provided to the driver during the driving time. Therefore, providing a visual distraction will complicate the visual task for the driver and this is not suitable for our goal.

\section{B. Proposed Method}

1) Protocol: The previous works showed that it is more suitable to measure implicitly the driver's SA in order to determine the costs and the benefits of the visual cues in the driving context. Based on this idea, we propose an experimental protocol to assess implicitly the driver's awareness of a pedestrian. This allows to identify the effects of the proposed AR-PCW system.

In order to assess the driver's awareness of a pedestrian (DAP), we create an ambiguous situation with pedestrians. A lead vehicle following task is proposed as a primary driving task. Indeed, the participant has to keep a constant distance with a lead vehicle. Therefore, the ambiguity happens when the driver enters to a situation where there is a pedestrian having intention to cross the road. This lead vehicle following task makes the experience more representative of typical driving situations and engages drivers so that the pedestrian would be more difficult to perceive or could be neglected by the driver.

The Fig. 3 shows the detail of a typical scenario with a pedestrian. The lead vehicle accelerates randomly when both vehicles enter into the situation with the pedestrian (from $100 \mathrm{~m}$ to pedestrian). At this point, the participant has to choose the right decisions between keeping distance with the lead vehicle and lifting up the pedestrian crossing.

In the experiment, the participant drives both times with two configurations of the system (noAR and AR configurations). In each driving, there are 23 pedestrians on road at different point. The positions, the appearances, and the behaviors of the pedestrians are randomly changed in different driving times. The driver is required to press a button placed on the steering wheel in order to indicate that he has noticed a pedestrian. The driver's performance in the lead vehicle following task and his reactions to avoid the accidents with the pedestrians are then measured in order to evaluate his situation awareness.

Before entering to the test, there is a practical session in which the instructor recommends and trains the driver to keep a distance about $50 \mathrm{~m}$ to the lead vehicle. When the participant feels familiar with the task, he can start the formal tests. There is no pedestrian in this session.

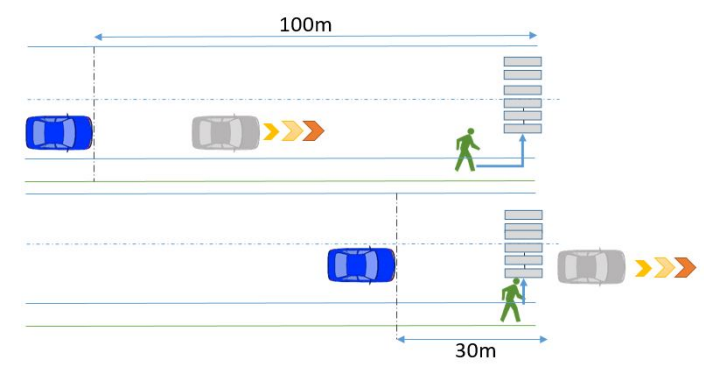

Figure 3: Typical scenario used in the experiment

2) Apparatus: The experiments are conducted on a driving simulator manufactured by [21]. This simulator interface is set up to be the most comfortable as possible in order to facilitate various conditions of the experiments. The simulator is configured as shown in the Fig. 4a. A projector screen is placed at $1.5 \mathrm{~m}$ in front of the driver with a real steering wheel mounted at a real comfortable position near the driver. The simulator is controlled by the driving engine SCANeR-Studio 
[21] which enables to create different driving scenarios as well as to record all necessary driving data.

We propose a transparency zone (higher opacity) rendered as a rectangle to simulate a combined HUD. This zone covers the center part of the driver's field of view. This transparency zone and the visual cues are supposed to create an impression of a HUD to the driver (see Fig. 4b).

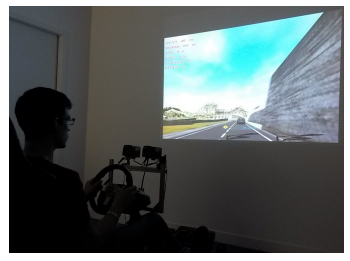

(a) Experimental Platform

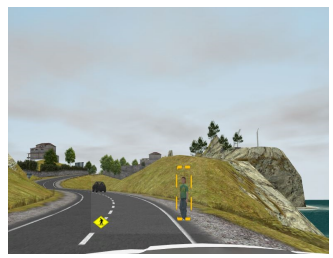

(b) Simulated HUD and AR cues in the virtual scene
Figure 4: Driving Simulator

3) Participants: The sample involved 27 subjects. Two of them did not finish the test due to the driver sickness of the simulator. All upcoming data refers to the 25 subjects who finished the test. All the participants were students, had at least three years licensed driving and was familiar with the simulator. Moreover, these participants had no disease about eye or physic at the moment of the test. They were ranged from 21 to 35 years old. 21 subjects are male and 4 subjects are female.

4) Dependent and independent variables.: In this experiment, independent variable includes participants, pedestrian situations and the lead vehicle's behaviors. We hypothesize that the system with its AR configuration would helps to enhance the driver's awareness three levels. Each level is then evaluated through the following outcome measures (dependent variables):

- Outcomes associated with the perception level: The Visual Reaction Time (VRT) is considered. The Visual Reaction Time is the moment (marked with the TTC) when the driver notices the pedestrian and presses the button. We expect that the AR cues will enhance the visibility of the pedestrian and the driver will notice the pedestrian sooner..

- Outcomes associated with the vigilance level: The Lead Vehicle Distance $(L V D)$ and the Accelerator Pedal Position $(A P P)$ are analyzed. With AR cues, we expect that the driver is more vigilant decelerates in the situation with pedestrian. Therefore, he will be less performing in the lead vehicle following task and the distance to the lead vehicle will be longer.

- Outcomes associated with the anticipation level: The number of time the driver brakes urgently is calculated. We hypothesize that the driver brakes urgently more often with the no cue compared to the driving with the AR cues.

\section{Results And Discussions}

In the result analysis step, we will consider the mean of each outcome over the participants and over the pedestrian situations. The differences between the outcomes from the drivings with two configurations (noAR and AR) will be exposed using the one-way Analysis of Variance (ANOVA ) tools [26].

\section{A. Outcomes associated to Perception Level}

a) Visual Reaction Times (VRT): This outcome is measured by the instant (calculated in Time-To-Collision) when the driver presses the button when he notices a pedestrian in front of the vehicle. Fourteen participants followed the recommendations and pressed the button when they noticed the pedestrian. The others just forgot the instructions and we could not get the $V R T$ data from these participants. Therefore, we had finally 14 participants for this result. The $V R T$ corresponds to the perception level of the DAP. The Fig 5 exposed the result of $V R T$ for 23 pedestrian situations whereas the Fig. 9a showed the mean values of $V R T$ of two configurations for all pedestrian scenarios. The ANOVA results confirmed there was a statistically significant difference between the noAR and AR configurations $(F(1,642)=23.46, p<0.05)$. With the noAR configuration, the driver noticed significantly lately the pedestrian when the vehicle is around $3 s$ of $T T C$ to the pedestrian and the driver noticed the pedestrian around for $4.5 \mathrm{~s}$ of $T T C$ with AR configuration. Regarding the Fig. 5, the $V R T$ values are found different between the pedestrian situations. This is perhaps due to the difference between the road situations, pedestrian's behaviors or the latency in button presses. However, in every situations, the driver noticed the pedestrian sooner with AR configuration.

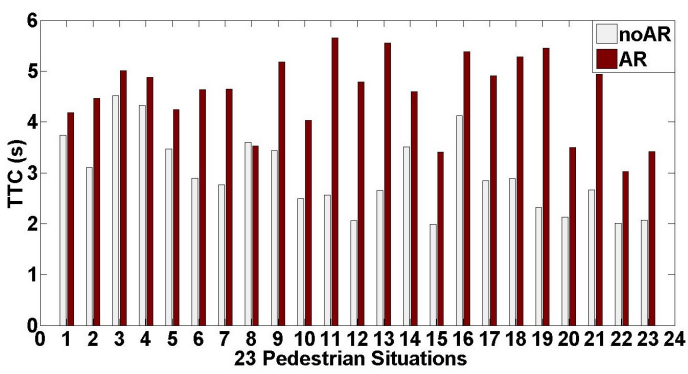

Figure 5: Mean of $V R T$ in each pedestrian situation. The result showed that, with the AR configuration (in dark color); the driver noticed earlier the pedestrian.

\section{B. Outcomes associated to Vigilance Level}

a) :

b) Accelerator Pedal Position (APP): In every pedestrian situation, the drivers were found to accelerate more aggressively when the cues were not provided (noAR configuration see Fig. 6). The ANOVA confirmed the difference of $A P P$ between two configurations $(F(1,1148)=18.47, p<$ $0.05)$. The test revealed that the driver pressed more deeply 
the accelerator pedal with noAR. The average of $A P P$ with noAR is equal to0.46 whereas with $\mathrm{AR}$ is equal to 0.23 . (see Fig. 9c).

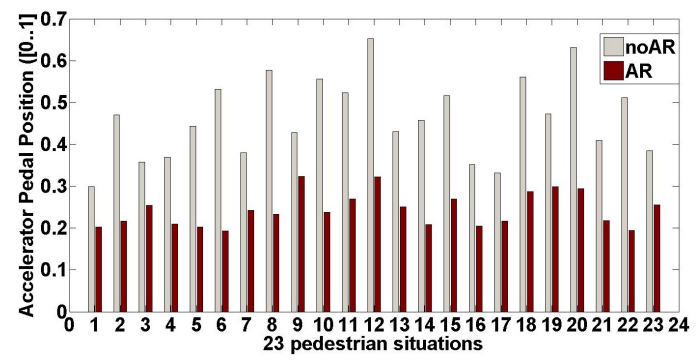

Figure 6: Mean of accelerator pedal position for the two configurations for each pedestrian situation. With noAR, the driver pressed more the accelerator pedal. The AR has effect on the driver's acceleration behaviors, the cues encourage the driver to slow down.

c) Lead Vehicle Distance (LVD): Graphically, head vehicle following task was performed better with the noAR, we observed that the driver kept a shorter distance to the lead vehicle compared to the AR configuration (see Fig. 7). The ANOVA results revealed a significant difference of $L V D$ between two configurations $(F(1,1148)=13.25, p<0.05)$. With the AR configuration, the $L V D$ was found in average at $118 \mathrm{~m}$ compared to $86 \mathrm{~m}$ with the noAR. The AR cues can somehow direct the attention of the driver to pedestrian and make him be more vigilant to pedestrians (see Fig. 9b).

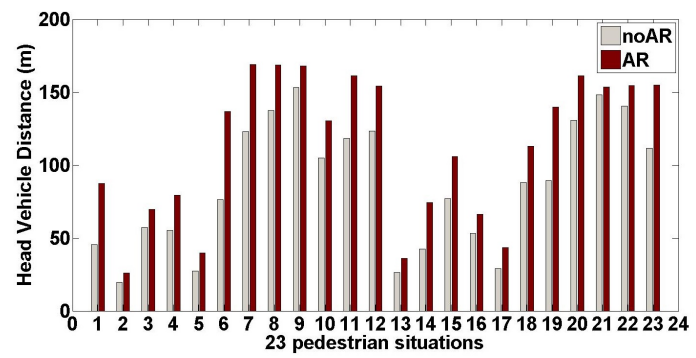

Figure 7: Lead Vehicle Distance for 23 pedestrian situations. In all pedestrian situations, the distance between two vehicles is greater with the AR configurations. The driver seems to pay less attention on the lead vehicle when the aids are on the pedestrian. The AR aids can direct the driver attention from the lead vehicle to the pedestrian.

\section{Outcomes associated to Anticipation Level}

a) Number of Urgent Braking Detected: A braking is considered to be urgent whenever the force applied on the pedal is detected to be over $200 N$ at a TTC smaller than $2 s$. In the total of all the driving test, 72 hard braking were detected with the noAR configuration, this number was found for 11 times with AR configuration (see Fig. 9d). The AR configuration is found having more influence on the driver's awareness.
The Fig. 8 showed the number of urgent braking detected in each pedestrian situation. With the noAR configuration, we found more often that the driver brakes urgently in front of the pedestrians. On the contrary, with the AR configuration, we found less the urgent braking. Indeed, the AR cues encouraged the driver to be attentive to pedestrian, more vigilant and finally it was found that the driver had a better anticipation in critical situations.

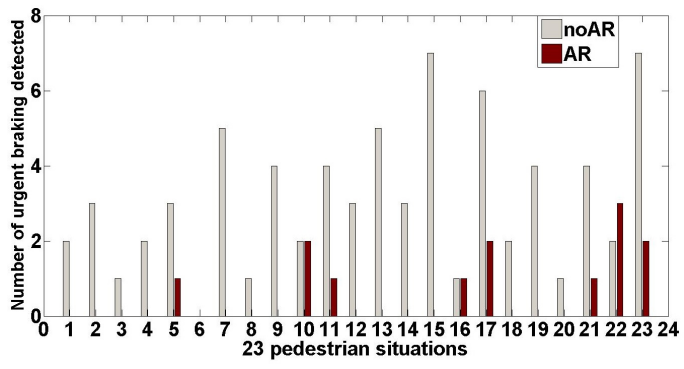

Figure 8: The Sum of number of times, the urgent braking was found in three configurations

\section{Subjective results analysis}

The Tab. I presented some our questionnaire results. The questionnaire was given after each driving with AR configuration. The results confirmed mostly the advantage of the AR configuration. Firstly all the participants feed-backed that they had driven like in the real life (the question Q01). This demonstrated that the drivers have felt comfortablywith the simulator. For the scenario difficulty, the lead vehicle following task was considered as quite difficult (question Q02), 20/25 participants have answered «difficult», and have commented that the difficulty was because the lead vehicle reacts too spontaneously. Moreover, 19/25 drivers answered that they had not tried to catch the lead vehicle (question Q04) and they had been aware of the pedestrians (answered as «normal» or «completely»).

For the questions concerning the cues, all the participants liked to have a HUD and the AR-PCWS on their cars (question Q05). 17/25 of participants preferred the bounding box compared to the pedestrian panel (question Q6). They argued that the bounding box helped them to identify where the pedestrian was, whereas the pedestrian panel did not and somehow forced them to brake and make them distracted.

\section{E. Discussion}

In this paper, three levels of the driver's awareness (perception, vigilance and anticipation) have objectively been assessed through the different outcome variables that represent the interaction between the driver and environment elements (lead vehicle and pedestrians). This protocol helps to measure objectively the driver's situation awareness without any distraction. The driver can drive normally during the test and is not interrupted for the questionnaire as using SAGAT technique. 


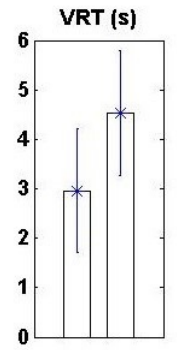

(a) $V R T$

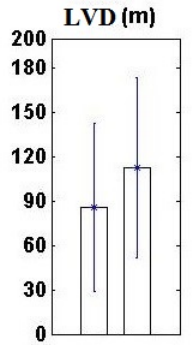

(b) $L V D$

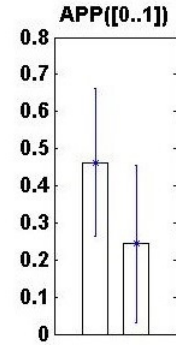

(c) $A P P$

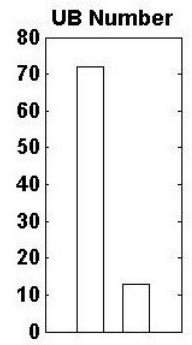

(d) Number of Urgent braking detected

Figure 9: Results in 23 pedestrian situations and 25 participants

\begin{tabular}{|c|c|c|}
\hline & Questions & Result \\
\hline \hline Q01 & Do you perform the driving like in real conditions? (Yes/No) & $25 / 25$ Yes \\
\hline Q02 & The driving task is difficult? (easy/normal/difficult/) & $20 / 25$ difficult \\
\hline Q03 & Are you try to catch the lead vehicle? (Yes/No) & $19 / 25$ No \\
\hline Q04 & Are you aware of the pedestrians?(completely/normal/not at all) & $4 / 25$ not at all \\
\hline Q05 & Would you like to use this combiner HUD ? (Yes/No) & $25 / 25$ Yes \\
\hline Q06 & Which cue do you prefer? (Bounding Box/Warning Panel) & $17 / 25$ Box \\
\hline
\end{tabular}

Table I: Some significant questions asked in the questionnaire

In the result, the AR aids were found to help the driver in the perception level, the driver perceived much sooner the pedestrian. We found the same conclusion with Schall et al. [25] that the aids could help the driver to perceive earlier the hazard. We found the same result as Rusch et al. [24], the visual aids could potentially direct the driver attention to the hazard.

Moreover, we found that the AR aids influenced the driver's vigilance. They did not focus only on the lead vehicle following task and were more aware of the pedestrians. The drivers did not brake urgently with the AR aids and were less aggressively driving in term of speed in the situation with pedestrian.

However, during the experiment, we have noticed that the driver's reactions varied a lot between the situations. In the results presented above, such as Visual Time Reaction, Accelerator Pedal Position, etc, the variances were really important. Indeed, the driver's awareness changed a lot in each pedestrian situation and a long the experiment. For the first pedestrians appearing on road, the drivers didn't care too much and they were less vigilant, but after certain times that pedestrians crossed the road, the drivers paid more attention and were more careful of that. Our technique of assessment of the driver's situation awareness necessities a wide range of situation in order to eliminate the repetition effect on the results. On the other side, the complexity of situations has to be the same because it also influences the results. Moreover, as human learns and anticipates fast, the driver's situation awareness is naturally improved during the experiment.

Since driving is a visual and motor control process, it is possible that the visual search demands associated with retrieving information from the displayed aids also degraded the driving task performance. In [10], the author highlighted that the automation human aids can cause comparable levels of distraction on driver navigation. However, It is also important to remark the different between conformal aids and non conformal aids. Indeed, the aids in their study is not conformal which can distract more than the conformal aids. The non-conformal aids (such as the warning panel) involve enclosed non-changing opaque rhombus shapes. These nonconformal aids therefore complicate the driver's ability to discriminate information about the targets (pedestrians) in which the aids highlighted. In contrast, the conformal aids (such as bounding box around the pedestrian in our work) were found that it did not produce any negative masking effects. After the experiment, the participants have commented that the conformal aids could enhance the awareness without distracting them.

We have found that the best strategy for building driving aids for achieving better situation awareness is to focus on specific problems where driving accidents are prevalent. Comparing to the related work, we provide an important aspect in designing a new ADAS interface that enhance the driver's situation awareness. Basically, these previous works considered subjectively the driver and his inattentive states such as sleepiness, drowsiness or distracted by hand phone which are helpful but not sufficient for the pedestrian accident purposes. The driver could be in normal state but if he underestimates the danger level of the situation, the accident can also happen. Therefore, our study is dedicated to the analysis of the driver interaction in a particular situation such as with pedestrians. Other critical situations have also been considered such as entering a intersection or complex danger scenarios [23], [2].

\section{CONClusion AND Future Work}

In this paper, an advanced driving assistance system using augmented reality cues has been proposed in the pedestrian 
safety context. The system highlights the pedestrian presence by a conformal bounding box. Moreover, it takes into account the time to collision and alert the driver in case there is a potential danger. It is expected to help the driver sooner identify the pedestrian and be aware of them. The study also highlighted the importance of validating a ADAS interface by assessing the driver's situation awareness while using the interface. Therefore, an experiment with 25 participants on a driving simulator was carried out to determine the effects of the visual cues on the driver in term of his/her awareness of a pedestrian.

AR cues may offer a promising mean to improve driver safety. During the experiment, we found out that the driver can achieve a higher perception level with the AR cues. The bounding box enhanced the visibility of the pedestrian, the driver noticed the bounding box where there was probably a pedestrian before noticing the pedestrian. The driver was also more vigilant with the AR cues (vigilance level). The cues somehow put the driver to a sensation that something could happen such as the pedestrian crossing. It encouraged the driver slowing down in situations. The results showed that the driver pressed less the accelerator pedal when the cues were displayed. In the anticipation level, the urgent braking was an indication to evaluate the driver's awareness. The preliminary result indicated that the driver braked urgently more often while driving without the aids.

However, these findings suggests that AR-ADAS merit further investigation. First of all, the study is based on a simulator in which the pedestrian detection is totally accurate which is not the case in reality. We will do a further analysis of the cues with different levels of pedestrian detection accuracy. Another experiments with new scenarios and more participants, and in real driving conditions using our intelligent vehicle platform ${ }^{1}$ will be carried out.

\section{ACKNOWLEDGMENT}

This work was carried out in the framework of the FUI18 SERA project, and the Labex MS2T which is funded by the French Government, through the program " Investments for the future" managed by the National Agency for Research (Reference ANR-11-IDEX-0004-02).

\section{REFERENCES}

[1] Amy L Alexander, Christopher D Wickens, and Thomas J Hardy. Synthetic vision systems: The effects of guidance symbology, display size, and field of view. Human Factors: The Journal of the Human Factors and Ergonomics Society, 47(4):693-707, 2005.

[2] G.S. Aoude, V.R. Desaraju, L.H. Stephens, and J.P. How. Driver behavior classification at intersections and validation on large naturalistic data set. Intelligent Transportation Systems, IEEE Transactions on, 13(2):724-736, June 2012.

[3] J Caird, W Horrey, and C Edwards. Effects of conformal and nonconformal vision enhancement systems on older-driver performance. Transportation Research Record: Journal of the Transportation Research Board, (1759):38-45, 2001.

[4] Alphonse Chapanis. Hazards associated with three signal words and four colours on warning signs. Ergonomics, 37(2):265-275, 1994

\footnotetext{
${ }^{1}$ Robotex, the national network of robotics platforms http: //equipex-robotex.fr/
}

[5] Vassilis Charissis and Stylianos Papanastasiou. Human-machine collaboration through vehicle head up display interface. Cognition, Technology \& Work, 12(1):41-50, 2010.

[6] Continental. Combiner head up display, 2015.

[7] Thomas A Dingus, Melissa C Hulse, Jonathan F Antin, and Walter W Wierwille. Attentional demand requirements of an automobile movingmap navigation system. Transportation Research Part A: General, 23(4):301-315, 1989.

[8] A Doshi, Shinko Yuanhsien Cheng, and M.M. Trivedi. A novel active heads-up display for driver assistance. Systems, Man, and Cybernetics, Part B: Cybernetics, IEEE Transactions on, 39(1):85-93, Feb 2009.

[9] Anup Doshi, Shinko Yuanhsien Cheng, and Mohan Manubhai Trivedi. A novel active heads-up display for driver assistance. Systems, Man, and Cybernetics, Part B: Cybernetics, IEEE Transactions on, 39(1):8593, 2009.

[10] Mary T Dzindolet, Linda G Pierce, Hall P Beck, and Lloyd A Dawe. The perceived utility of human and automated aids in a visual detection task. Human Factors: The Journal of the Human Factors and Ergonomics Society, 44(1):79-94, 2002.

[11] M. R. Endsley. Toward a theory of situation awareness in dynamic systems. Human Factors: The Journal of the Human Factors and Ergonomics Society, 37(1):32-64, 1995.

[12] Mica R Endsley. Measurement of situation awareness in dynamic systems. Human Factors: The Journal of the Human Factors and Ergonomics Society, 37(1):65-84, 1995.

[13] Y. Fukagawa and K. Yamada. Estimating driver awareness of pedestrians from driving behavior based on a probabilistic model. In Intelligent Vehicles Symposium (IV), 2013 IEEE, pages 1155-1160, June 2013.

[14] Elisa Drelie Gelasca, Danko Tomasic, and Touradj Ebrahimi. Which colors best catch your eyes: a subjective study of color saliency. In Fisrt International Workshop on Video Processing and Quality Metrics for Consumer Electronics. Citeseer, 2005.

[15] Kenneth W Gish and Loren Staplin. Human factors aspects of using head up displays in automobiles: A review of the literature. 1995.

[16] Paul Green. The 15-second rule for driver information systems. In Proceedings of the ITS America Ninth Annual Meeting, 1999.

[17] R Ma and David B Kaber. Situation awareness and driving performance in a simulated navigation task. Ergonomics, 50(8):1351-1364, 2007.

[18] Shane B McLaughlin, Jonathan M Hankey, and Thomas A Dingus. A method for evaluating collision avoidance systems using naturalistic driving data. Accident Analysis \& Prevention, 40(1):8-16, 2008.

[19] Mobileye. Mobileye pedestrian collision warning (pcw). Online, 2015.

[20] B. Metz N. Schomig. Three levels of situation awareness in driving with secondary tasks. Safety Science, 56(0):44 - 51, 2013. Situation Awareness and Safety.

[21] Oktal. Driving simulation engine scaner http://www.scanersimulation.com, 2015.

[22] Hye Sun Park and Kyong-Ho Kim. Driver-view based augmentedreality hud system: concept and background. In 19th ITS World Congress, 2012.

[23] Marina Plavšić, Gudrunk Klinker, and Heiner Bubb. Situation awareness assessment in critical driving situations at intersections by task and human error analysis. Human Factors and Ergonomics in Manufacturing \& Service Industries, 20(3):177-191, 2010.

[24] Michelle L. Rusch, Mark C. Schall Jr., Patrick Gavin, John D. Lee, Jeffrey D. Dawson, Shaun Vecera, and Matthew Rizzo. Directing driver attention with augmented reality cues. Transportation Research Part F: Traffic Psychology and Behaviour, 16(0):127 - 137, 2013.

[25] Mark C Schall Jr, Michelle L Rusch, John D Lee, Jeffrey D Dawson, Geb Thomas, Nazan Aksan, and Matthew Rizzo. Augmented reality cues and elderly driver hazard perception. Human Factors: The Journal of the Human Factors and Ergonomics Society, 55(3):643-658, 2013.

[26] Laerd Statistics. One-way anova in spss. Retrieved December, 21:2013, 2013.

[27] Nicholas J Ward and Andrew Parkes. Head-up displays and their automotive application: An overview of human factors issues affecting safety. Accident Analysis \& Prevention, 26(6):703-717, 1994.

[28] WHO. World health organization, 2013. global status report on road safety supporting a decade of action. 2013. 